\title{
Intelligent Transportation Systems and Greenhouse Gas Reductions
}

\author{
Matthew J. Barth ${ }^{1} \cdot$ Guoyuan Wu ${ }^{1} \cdot$ Kanok Boriboonsomsin ${ }^{1}$
}

Published online: 26 July 2015

(C) Springer International Publishing AG 2015

\begin{abstract}
The field of Intelligent Transportation Systems (ITS) has witnessed significantly increased activity in recent years, with the application of modern control, communications, and information technology to vehicles, roadway infrastructure, and traffic information systems. The primary objectives of ITS have been focused primarily on improving safety and increasing mobility and associated transportation efficiency. In addition, it is now well known that ITS technology can be used to reduce transportation-related environmental impacts. These environmental impacts include pollutant emissions that lead to poor air quality, as well as energy consumption and greenhouse gas (GHG) emissions. Over the years, we have seen many ITS programs focusing on safety and mobility that end up also having significant environmental benefits. These benefits range widely due to a number of variables. However in recent years, a number of ITS research programs have emerged that are specifically designed to minimize the environmental impacts of transportation. In this manuscript, we briefly outline how the different areas of ITS can impact GHG emissions and then describe a number of recent ITS programs that are specifically aimed at reducing energy and environmental impacts. These environmental ITS programs
\end{abstract}

This article is part of the Topical Collection on Transportation

Matthew J. Barth

barth@cert.ucr.edu

Guoyuan $\mathrm{Wu}$

gywu@cert.ucr.edu

Kanok Boriboonsomsin

kanok@cert.ucr.edu

1 Bourns College of Engineering-Center for Environmental Research and Technology, University of California, Riverside, 1084 Columbia Avenue, Riverside, CA 92507, USA typically show energy and emission reductions on the order of $5-15 \%$.

Keywords Intelligent Transportation Systems · Safety · Mobility · Environmental impacts · Greenhouse gas emissions · Energy consumption · Environmental ITS programs

\section{Introduction}

Due to their potential to improve roadway safety, reduce traffic congestion, and enhance the mobility of people and goods, Intelligent Transportation Systems (ITS) have generated considerable enthusiasm in the transportation community. In addition to safety and mobility, we are now seeing that ITS can play a major role in reducing both pollutant and greenhouse gas (GHG) emissions, as well as reducing energy consumption. It is commonly understood that the transportation sector is responsible for nearly a third of the emitted GHG emissions (see, e.g., [1]). In order to reduce these GHG contributions, much of the focus has been put on (1) improving overall vehicle fuel economy (e.g., using more efficient innovative drivetrains, reducing weight while maintaining safety); (2) using less carbon-intensive fuels (e.g., ethanol, electricity); and (3) managing travel demand (e.g., roadway tolling) and shifting travel onto nonmotorized modes. In addition, we are now seeing that ITS can be applied as the fourth key component, namely to improve the efficiencies of transportation system operations, thereby reducing overall GHG emissions.

By its nature, ITS consists of a wide variety of technologies and applications, as described in the US National Intelligent Transportation System Architecture [2]. In general, they can be categorized into three major target areas: Vehicle Systems, Traffic Management Systems, and Travel Information 
Systems. We briefly introduce these general areas below, describing how they potentially can reduce GHG emissions.

\section{Vehicle Systems}

Vehicles are now taking advantage of modern control systems, faster on-board processors, and wireless communications to provide features that greatly improve their performance. Examples of emerging vehicle systems include the following:

Longitudinal Assistance Systems are concerned with, for example, preventing front-end and rear-end collisions. On-board radar, LiDAR, and computer vision technology are now being applied to monitor headways between vehicles, and by providing feedback to the vehicle's braking system, collisions can be reduced. These sensors are also being used for Adaptive Cruise Control (ACC) systems that not only allow a driver to select a desired speed but also allow setting the following distance. With the addition of wireless communication capabilities, ACC can evolve into Cooperative Adaptive Cruise Control (CACC), which is now emerging as a popular area of research.

Lateral Assistance Systems are being designed to improve the performance of vehicles during lane changes, merges, or any kind of turning movement. Computer vision technology and other sensors coupled with wireless communications are being deployed to provide lane departure warnings and to warn drivers of pending lateral collisions. Both longitudinal and lateral control systems are now leading us down the path towards partial and full automation, where vehicles will automatically drive themselves with little input from the drivers.

Wireless Communications Systems are now being deployed in vehicles in a number of different ways. Cellular communication technology is already playing a large role in fleet management applications and vehicle monitoring. In addition, dedicated short-range communication (DSRC) radios will likely be deployed to enable vehicle-to-vehicle (V2V), vehicle-to-infrastructure (V2I), and infrastructure-to-vehicle (I2V) applications that are primarily focused on improving safety. However, mobility and environmental applications will also likely emerge that take advantage of this "connected vehicle" technology.

The majority of these ITS-related vehicle systems are primarily focused on safety, resulting in a reduction of the number of accidents that occur on our roadways. As such, accident-related congestion will also be reduced, and traffic will flow more smoothly. This should result in a significant GHG emissions benefit as any type of congestion mitigation is beneficial from an environmental perspective. When vehicles crawl in slow, stop-and-go traffic, their energy consumption and GHG emissions per unit distance are significantly higher compared to traveling at steady, moderate speeds (i.e., less acceleration/deceleration at speeds around $80 \mathrm{kph}$ for most light-duty vehicles, see [3]). However, it is important to note that traveling at very high speeds (e.g., $100 \mathrm{kph}$ and above) will increase GHG emissions due to aerodynamic drag effects.

\section{Traffic Management Systems}

Over the last several decades, the total amount of driving (as measured in vehicle miles traveled or VMT) has grown significantly, contributing to severe roadway congestion in many urban areas. Building additional infrastructure to handle the increase in travel demand is not always possible. However, there are a number of ITS-based Traffic Management System solutions that can help mitigate congestion:

Traffic Monitoring Systems are improving with better sensor technology, more reliable communication channels, and more advanced information processing capability. In addition to providing transportation managers with better real-time information, new data processing techniques are being developed to estimate traffic flow, density, and speed, as well as other microscopic traffic parameters. This real-time traffic information can be used for better traffic system management and for individual drivers choosing alternative routes, resulting in a reduction of congestion.

Traffic Incident Management techniques are important tools in terms of early detection and rapid removal of incidents (i.e., accidents, disabled vehicles, etc.) so that normal traffic operations recover as quickly as possible. Integrated Corridor Management techniques are applicable in a cooperative way to both freeway networks (e.g., innovative ramp metering) and to signalized arterial networks (e.g., advanced signal timing algorithms). These techniques are designed to keep traffic flowing as smoothly as possible through the corridor, greatly reducing the amount of idling.

Travel Demand Management is another critical element of traffic management. By reducing the number of vehicles on a congested roadway or spreading out the peak of traffic volume through pricing and other techniques, traffic flow will improve, resulting in lower GHG emissions.

The general goal of traffic management is to take full advantage of capacities of existing roadway infrastructure, thus keeping traffic flowing smoothly at moderate speeds. As such, this will have a large impact in reducing energy consumption and GHG emissions. Traffic management system strategies go even further by reducing the number of vehicles in the 
transportation system, thereby reducing the total contributions of GHG emissions.

\section{Travel Information Systems}

To make things more convenient to drivers, a wide variety of information systems for travelers have recently evolved. Examples of this technology include the following:

Route Guidance Systems have greatly improved in recent years in a variety of forms including on-board, off-board, and smartphone-based systems. These navigation systems now use geographic and real-time traffic information and can select optimal routes in a roadway network from specific origins to specific destinations. These systems attempt to minimize some criteria, such as travel time, travel distance, or even GHG emissions.

Geo-Location Systems are typically coupled with route guidance systems to allow users to find specific locations, cutting down on excessive driving (e.g., searching for a gasoline filling station, open parking space, etc.).

Electronic Payment Systems are becoming increasingly prevalent, allowing for the payment of tolls and fees without stopping the vehicle to complete the financial transaction.

All of these different systems are convenient for the traveler; in addition, they also can have a significant benefit in reducing GHG emissions. As described above, a route guidance system will cut back on unnecessary travel that may occur when a driver gets lost or chooses a long, out-of-theway path. En route driver information is also beneficial by eliminating the additional GHG emissions associated with driving around, searching for these specific goals. Electronic payment systems eliminate the need for a driver to decelerate the vehicle, idle while a manual transaction takes place, followed by an acceleration that gets the vehicle back up to a desired speed. If this payment can occur without slowing down, GHG emissions are reduced.

All of these ITS areas described above have the potential for indirectly reducing GHG emissions through improvements in safety, mobility, and driver convenience. The actual GHG emission reductions will vary significantly, depending on a large number of factors. These factors include, for example, vehicle fleet mix, traffic volume and dynamics, and the type of roadway network and infrastructure. A variety of these ITS applications have been designed and modeled, and several have been piloted and implemented in different locations. In order to determine the overall effectiveness of these applications, different performance measures are estimated, usually mobility measures such as improvements in average travel time or reductions of network delay. In some cases, environmental factors are also estimated. One straightforward method that is often used is to monitor the changes in average travel times (and therefore average speeds), normalized by the total amount of traffic. In general, if overall traffic conditions move from a congested regime (e.g., average speeds below $30 \mathrm{mph}$ ) to a less congested regime (speeds greater than $30 \mathrm{mph}$ ), then, emission reductions per vehicle can be estimated using speedemission factor curves [3]. As an example, traffic smoothing techniques have been shown in [4] to result in a $10-20 \% \mathrm{CO}_{2}$ reduction.

One concern that has emerged with any type of ITS implementation is the potential for induced travel demand. It has been shown that if roadway capacity is increased (e.g., building additional roads or adding more lanes), typically the amount of vehicle miles traveled (VMT) also increases (e.g., see [5]). In terms of ITS, the hypothesis is that if the overall mobility improves, then potentially, VMT will increase. To date, there have not been any definitive studies showing this effect. However, current research is examining how automated vehicles may affect travel demand (see, e.g., [6]). Preliminary results show that with a modest introduction of automated vehicles, $5 \%$ increase in VMT may occur. Nevertheless, the general effects of ITS on travel demand need to be studied in greater detail.

In addition to the ITS applications described above, a number of ITS research programs have emerged that have been specifically designed to minimize transportation GHG emissions. The remainder of this manuscript describes several of these recent ITS programs that are targeting the energy and environmental impacts of transportation.

\section{Recent Environmental ITS Research Programs in the USA}

In the last decade, the U.S. Department of Transportation (USDOT) has initiated a variety of environmentally focused ITS research programs. Many of these are part of the Federal Highway Administration Exploratory Advanced Research program (FHWA EAR, see [7]). As an example in the ITS vehicle systems area, researchers at the University of California (UC), Berkeley, have investigated both ACC and CACC and their impacts on mobility and the environment [8]. Furthermore, as an example in the area of traffic management systems, there is an on-going EAR project on Advanced Traffic Signal Control Algorithms, where several algorithms have been developed specifically for reducing energy use and emissions (see, e.g., [9]). Results from these studies show GHG emission reductions in the range of 5 to $10 \%$.

In addition to the EAR program, the USDOT has a major University Transportation Centers program (UTC, see [10]), where university faculty, staff, and students across the USA work on a number of advanced transportation research projects. There are now a number of UTCs that have a focus on 
sustainability, and several of those are investigating the environmental impacts of ITS (see, e.g., [11, 12]). Some example projects from these centers include examining eco-driving techniques and freight signal priority for heavy-duty vehicles (typically 5-8\% reductions, see, e.g., [13]) and how variable speed limits can be used to reduce transportation energy consumption and improve vehicle mobility [14].

As another major effort, the USDOT has a long-term research program in connected vehicles. One of the foundational elements of the connected vehicle research effort in the environment area is the Applications for the Environment: Realtime Information Synthesis (AERIS) program [15]. Using vehicle-to-vehicle (V2V), vehicle-to-infrastructure (V2I), and infrastructure-to-vehicle (I2V) communications, the goal of the AERIS program is to design ITS applications that aim to reduce energy consumption and emissions. Several AERIS operational concepts have been developed, including (1) Eco-Signal Operations; (2) Eco-Lanes; (3) Dynamic Low Emissions Zones; (4) Support for Alternative Fuel Vehicle (AFV) Operations; (5) Eco-Traveler Information; and (6) Eco-Integrated Corridor Management (ICM).

Early in the AERIS program, there were several initial exploratory research projects carried out by a variety of researchers in the USA. These projects investigated a number of new concepts across the different areas of ITS, including

\section{Vehicle Systems}

- Developing and Evaluating Intelligent Eco-Drive Applications, carried out by researchers at Virginia Tech [16]. This project focused on the development of an eco-ACC system and evaluated the network-wide impacts of such systems for different levels of market penetration and network configurations. Results showed GHG emission reductions up to $49 \%$.

- Assessment, Fusion, and Modeling of Commercial Vehicle Engine Control Unit Data, carried out by Calmar Telematics (with UC, Riverside) [4]. This project investigated the use of real-time, on-board data to calculate environmental performance measures based partly upon the team's Comprehensive Modal Emissions Model (CMEM).

\section{Traffic Management Systems}

- Eco-Speed Control Using V2I communications also carried out by Virginia Tech [16]. In this project, innovative eco-adaptive signal control algorithms were developed and tested. These systems were modeled using traffic simulation software and tested for different roadway configurations.

- Eco-Friendly Intelligent Transportation Systems (ECOITS), carried out by UC, Riverside [17]. This project is built on previous ECO-ITS research in predicting secondby-second fuel consumption and tailpipe emissions for different environmental ITS applications and strategies, such as advanced traffic signalization.

\section{Travel Information Systems}

- An Evaluation of Likely Environmental Benefits of Lowest Fuel Consumption Route Guidance in the BuffaloNiagara Metropolitan Area, carried out by the University at Buffalo [18]. This study assessed the likely environmental benefits of a new application for an environmentally optimized route guidance system for medium-sized metropolitan areas. Results showed GHG emission reductions in the range of $5-10 \%$.

After these initial exploratory projects, the AERIS operational concepts were further developed and defined. The main AERIS applications that were evaluated in detail are the EcoTraffic Signal Operational concept, including the applications of Eco-Approach and Departure at Signalized Intersections, Eco-Traffic Signal Timing, and Eco-Traffic Signal Priority, as well as the Eco-Lanes Operational concept, which includes the applications of Eco-Speed Harmonization and EcoCooperative Adaptive Cruise Control. These applications are described in further detail below.

\section{Eco-Traffic Signal Operations}

There has been a good deal of research in eco-traffic signal operations around the world. These eco-signal operations consist of the use of ITS technologies to decrease fuel consumption and GHG emissions on arterial roadways by reducing idling, the number of stops, and unnecessary accelerations and decelerations at signalized intersections. Prior to the emergence of connected vehicles, eco-traffic signal operations were primarily focused on synchronizing traffic signals to improve traffic flow and thereby reducing GHG emissions. By taking advantage of $\mathrm{V} 2 \mathrm{I} / \mathrm{I} 2 \mathrm{~V}$ communications, a number of other signalization strategies emerge, three of which are described below.

Eco-Approach and Departure at Signalized Intersections This application uses wireless data communications sent from the traffic signal controller to connected vehicles to encourage "green" approaches to signalized intersections. ${ }^{1}$ This includes broadcasting Signal Phase and Timing (SPaT) data and a Geographic Information Description (GID) or MAP data. Vehicle status messages, sent from nearby vehicles using V2V

\footnotetext{
${ }^{1}$ Note that the eco-approach and departure application is also similar to the application in Europe known as "Green Light Optimal Speed Advisory" (GLOSA).
} 
communications, are also considered by the application. Upon receiving this information, an on-board processor can then calculate an optimal speed trajectory to approach, pass through, and depart from a signalized intersection. This speed trajectory can be provided as advice to the driver through a human-machine interface, or directly to the vehicle's longitudinal controller (such as the ACC system). The general strategy is to have the vehicle change its speed to pass the upcoming traffic signal on green or to decelerate to a stop in the most eco-friendly manner. This application also considers a vehicle's acceleration as it departs from a signalized intersection and engine start-stop technology as a vehicle is stopped at a traffic signal. In terms of results, the AERIS program has shown that there is 2 to $7 \%$ energy savings for all vehicles and that the application is less effective when the corridor is congested (see, e.g., $[19,20]$ ).

Eco-Traffic Signal Timing This application is similar to current adaptive traffic signal systems; however, the application's objective is to optimize traffic signals for the environment. The application collects data from vehicles, such as vehicle location, speed, GHG, and other emissions using connected vehicle technologies. It then processes these data to develop signal timing strategies that are focused on reducing fuel consumption and overall emissions at an intersection, along a corridor, or for a region. The application evaluates traffic and environmental parameters at each intersection in realtime and adapts so that the traffic network is optimized using available green time to serve the actual traffic demands while minimizing the environmental impact. In terms of results, the AERIS program has shown that there is 1 to $5.5 \%$ energy savings and that the application is effective in most conditions other than full saturation [21].

Eco-Traffic Signal Priority This application allows either transit or freight vehicles approaching a signalized intersection to request signal priorities. This application considers the vehicle's location, speed, vehicle type (e.g., alternative fuel vehicles) and associated GHG and other emissions to determine whether priority should be granted. Information collected from vehicles approaching the intersection, such as a transit vehicle's adherence to its schedule, or the number of passengers on the transit vehicle may also be considered in granting priority. If priority is granted, the traffic signal would attempt to hold the green or terminate the red on the approach to facilitate the transit/freight vehicle passing through the intersection without stopping. This application does not consider signal preemption, which is reserved for emergency response vehicles. In terms of results, the AERIS program has shown that there is 1 to $4 \%$ energy savings for all freight vehicles and 1 to $2 \%$ savings for transit vehicles [21].

\section{Eco-Lanes Operations}

Another environmentally focused connected vehicle application is the "Eco-Lane" concept. This concept uses connected vehicle technologies to decrease fuel consumption and GHG as well as other criteria air pollutant emissions on highways by reducing congestion and unnecessary accelerations/decelerations, improving traffic flow, and encouraging greener driving behavior. It is envisioned that this could occur either on a single lane or across multiple lanes on a freeway. This EcoLane concept can consist of a number of different applications that are specifically set up for a freeway scenario:

- Eco-Lanes Management: This application supports the operation of dynamic eco-lanes, including establishing qualifications for entering the lanes, defining activation periods, or geo-fencing the eco-lanes' boundaries.

- Eco-Speed Harmonization: The Eco-Speed Harmonization application assists in smoothing traffic flow, reducing unnecessary stops and starts, and maintaining consistent speeds, thus reducing fuel consumption, GHGs, and other emissions on the roadway. This can be accomplished typically through variable speed limits.

- Eco-Cooperative Adaptive Cruise Control: The EcoCooperative Adaptive Cruise Control application allows individual drivers to opt into applications that take advantage of adaptive cruise control capabilities along with $\mathrm{V} 2 \mathrm{~V}$ communications designed to minimize vehicle accelerations and decelerations for the benefit of reducing fuel consumption and vehicle emissions.

- Eco-Ramp Metering: The Eco-Ramp Metering application determines the most environmentally efficient operation of traffic signals at freeway on-ramps to manage the rate of vehicles entering a freeway.

- Connected Eco-Driving: The Connected Eco-Driving application provides customized real-time driving advice to drivers so that they can adjust their driving behavior (e.g., speed of travel, acceleration and deceleration rates) to save fuel and reduce emissions.

- Multi-Modal Traveler Information: The Multi-Modal Traveler Information application provides pre-trip and en route multi-modal traveler information to encourage environmentally friendly transportation choices.

In the USA, the AERIS program's Eco-Lanes concept modeling was focused only on the eco-speed harmonization and eco-cooperative adaptive cruise control application [22]. Using a sophisticated set of simulation modeling tools, it was shown that under the assumption of $100 \%$ penetration rate, eco-speed harmonization could result in up to $12 \%$ energy savings, but with an $8 \%$ reduction in mobility (i.e., longer travel times). However, when tuned so that mobility was unaffected, then the environmental benefits were around $5 \%$. On 
the other hand, eco-cooperative adaptive cruise control was shown to provide upwards of $30 \%$ energy savings for a generic freeway segment. When applied to a regular freeway with on- and off-ramps, the savings were around $15 \%$.

\section{Recent Environmental Research Programs in the European Union}

In 2011, the European Union (EU) set up Working Group for Clean and Efficient Mobility (WG4CEM) as part of the iMobility Forum with the aim to identify the most promising ITS solutions for clean and efficient mobility. Part of the effort of this group is to provide a priority list of recommendations of ITS environmental projects as well as a roadmap illustrating the pathways of research. This European working group consists of members from road authorities/road operators, road users, and automotive and information and communications technology (ICT) industry stakeholders interested in clean and efficient mobility. Connected vehicles and infrastructure were a subset of the proposed solutions. The results of this effort can be found in [23].

In this section, we only highlight some of the major ITS projects aimed at reducing GHG emissions, and in particular, those involving connected vehicles. Many of these projects were funded by various sources, including the European Commission (EC), the European Commission Directorate General for Communications Networks, Content and Technology (DG-CONNECT), the European Union's Seventh Framework Program for Research (FP7), and the EU Competitiveness and Innovation Framework Program and its Policy Support Program (CIP-PSP). These programs are categorized into the three major ITS areas.

\section{Vehicle Systems}

The EU carried out a major program in connected vehicles called "eCoMove" which was focused on "cooperative mobility systems and services for energy efficiency." This program examined the latest $\mathrm{V} 2 \mathrm{I} / \mathrm{I} 2 \mathrm{~V}$ and $\mathrm{V} 2 \mathrm{~V}$ communication technologies and created an integrated solution comprising eco-driving support and eco-traffic management to tackle the main sources of energy waste by passenger and commercial vehicles. The eCoMove project targeted three main causes of avoidable energy use by road transport to bring fuel wastage to a minimum: (1) inefficient route choice; (2) inefficient driving performance; and (3) inefficient traffic management and control. Overall, the eCoMove project showed fuel savings of around 10 to $20 \%$ for eco-driving and approximately $10 \%$ savings for traffic signal operations [24].

Another important project was the ECOSTAND project, which was a joint EU-Japan-US task force on the development of a standard methodology for determining the impacts of ITS on energy efficiency and carbon dioxide $\left(\mathrm{CO}_{2}\right)$ emissions. The overall goal was to provide support for an agreement between three regions - the European Union, Japan, and the USA — on a framework for a common assessment methodology for quantifying the impacts of ITS on energy efficiency and $\mathrm{CO}_{2}$ emissions [25].

Currently, Compass4D is an on-going multi-city project in Europe that is focused on advanced vehicle cooperative system deployment to improve road safety and energy efficiency and to reduce congestion. This 3-year EU co-funded project (started in January 2013) is deploying three services in seven cities (Bordeaux, Copenhagen, Helmond, Newcastle, Thessaloniki, Verona, and Vigo), in order to prove the concrete benefits of cooperative systems for citizens, city administrations, and companies. Compass4D is piloting three main services: red light violation warning, road hazard warning, and energy efficient intersection. These services are in place for 1 year on 334 vehicles, including buses, taxis, emergency vehicles, and private cars, reaching more than 550 users [26]. Results on environmental impacts are pending.

\section{Traffic Management Systems}

The "Co-operative Systems for Sustainable Mobility and Energy Efficiency" or COSMO project adopted a system-wide approach to the assessment of energy efficiency, measuring the effect of a range of innovative traffic management systems not only on fuel consumption and emissions of vehicles but also on the energy used to operate road-side equipment. The aim was to provide a practical demonstration of the effectiveness of the systems and to assess their impact under realistic conditions. The principal result was a set of validated specifications covering their operational, technical, business, and organizational characteristics. The COSMO test sites were in Salerno, Vienna, and Gothenburg and ran from 2010 to 2013 [27]. In general, $\mathrm{CO}_{2}$ emission reductions were shown on the order of 5-15\%.

The "Co-operative Networked Concept for Emission Responsive Traffic Operations" or ConCERTO project was carried out from 2010 to 2013 and used highly sophisticated environment tools to develop next-generation technologies to reduce motor vehicle emissions based on real-time emission measurements [28].

The CARBOTRAF project is currently underway and is focusing on developing "A Decision Support System for Reducing $\mathrm{CO}_{2}$ and Black Carbon Emissions by Adaptive Traffic Management." The overall goal is to develop tools for adaptively influencing traffic in real-time to reduce $\mathrm{CO}_{2}$ and black carbon emissions caused by road transport in urban and interurban areas [29]. 


\section{Traveler Information Systems}

In 2012, the "Assessment Methodologies for ITS in multimodal transport from User Behavior to $\mathrm{CO}_{2}$ reduction" (AMITRAN) program began and ran until 2014. This program developed a framework for the evaluation of the effects of ITS measures in traffic and transport on energy efficiency and $\mathrm{CO}_{2}$ emissions [30].

The EcoGem or "Cooperative Advanced Driver Assistance System for Green Cars" project was carried out from 2010 to 2013 and aimed to provide efficient connected vehicle solutions to support electric vehicles (EVs). The goal was to design and develop an EV-oriented, highly innovative Advanced Driver Assistance System (ADAS) equipped with suitable monitoring, learning, reasoning, and management capabilities that would help increase the EV's autonomy and energy efficiency [30].

\section{Conclusions}

In this manuscript, we highlighted state-of-the-practice ITS programs that are environmentally beneficial, spanning the three main areas of ITS, including Vehicle Systems, Traffic Management Systems, and Traveler Information Systems. For the last several decades, many of these ITS programs have been targeted at improving safety and reducing congestion, with the secondary goals of reducing fuel consumption and lowering pollutant and GHG emissions. It is clear that specific environmental benefits can be maximized when these different ITS applications are "tuned" so that emissions and fuel consumption are reduced.

Furthermore, it is important to point out that there is not a single ITS technology solution that has a far-reaching impact at reducing GHG emissions. Each of the ITS environmentally beneficial applications described in this manuscript typically have GHG reductions in the range of 5-15\%. However, most of these applications are additive; therefore, greater benefits may be achieved when a multitude of environmentally friendly ITS programs are put into place. It is clear that as more ITS programs are deployed, environmental impact assessments must be performed.

Even with these ITS operational improvements that result in reduced emissions, we must also be concerned about any potential induced demand effects. Again, this needs to be modeled and measured as new ITS applications are deployed. It may be that to counteract these effects, the ITS programs may have to be coupled with some specific travel demand management measures.

Acknowledgments The authors would like to acknowledge various sources for the materials in this chapter, both in research support and funding, including the University of California Transportation Center
(UCTC), the National Center for Sustainable Transportation (NCST), the California Department of Transportation, and the USDOT's AERISconnected vehicle research program. The contents of this chapter reflect the views of the authors and do not necessarily indicate acceptance by the sponsors.

\section{Compliance with Ethics Guidelines}

Conflict of Interest Matthew J. Barth, Guoyuan Wu, and Kanok Boriboonsomsin declare that they have no conflict of interest.

Human and Animal Rights and Informed Consent This article does not contain any studies with human or animal subjects performed by any of the authors.

\section{References}

1. [USEPA, 2015] U.S. Environmental Protection Agency. U.S. greenhouse gas inventory report: 1990-2013, see http:/www.epa. gov/climatechange/ghgemissions/usinventoryreport.html. 2015.

2. U.S. Department of Transportation. Intelligent transportation systems joint program office. National ITS Architecture, see http:// www.iteris.com/itsarch/. Accessed May 2015.

3. Barth M, Boriboonsomsin K. Real-world carbon dioxide impacts of traffic congestion. Transp Res Rec J Transp Res Board. 2008;2058: 163-71.

4. Barth M, Mandava S, Boriboonsomsin K, Xia H. Dynamic ECOdriving for arterial corridors. IEEE Forum on Integrated and Sustainable Transportation System (FISTS), June. pp. 182-8.

5. Noland R. Relationships between highway capacity and induced vehicle travel. Transp Res A Policy Pract. 2001;34:47-72

6. Gucwa M. Mobility and energy impacts of automated cars. Proceedings of the Automated Vehicles Symposium, San Francisco, July 2014

7. U.S. Department of Transportation, Federal Highway Administration Exploratory Advanced Research Program, see http://www.fhwa.dot.gov/advancedresearch. 2015.

8. Milanes V, Shladover SE, Spring J, Nowakowski C, Kawazoe H, Nakamura M. Cooperative adaptive cruise control in real traffic situations. IEEE Trans Intell Transp Syst. 2014;15(1):296-305

9. Hao P, Wu G, Boriboonsomsin K, Barth M. Developing a framework of eco-approach and departure application for actuated signal control. IEEE Intelligent Vehicles Symposium. 2015 (accepted).

10. U.S. Department of Transportation. University transportation centers program, see http://www.rita.dot.gov/utc/home. 2015.

11. National Center for Sustainable Transportation, see http://ncst. ucdavis.edu/. 2015.

12. Mid-Atlantic Transportation Sustainability Center, see http://www. matsutc.org/. 2015.

13. Kari $\mathrm{D}, \mathrm{Wu} \mathrm{G}$, Barth $\mathrm{M}$. Eco-friendly freight signal priority using connected vehicle technology: a multi-agent systems approach. Proceedings of the 2014 I.E. Symposium on Intelligent Vehicles. Dearborn, Michigan; June, 2014.

14. Ahn K, Rakha H. Ecolane applications. Transp Res Rec J Transp Res Board. 2014;2427(1):41-53.

15. U.S. Department of Transportation. Applications for the Environment: Real-Time Information Synthesis (AERIS) research program, see http://www.its.dot.gov/aeris/. 2015.

16. Virginia Tech Transportation Institute, see http://www.its.dot.gov/ aeris/pdf/EcoDriving.pdf. 2011.

17. University of California, Riverside, see http://ntl.bts.gov/lib/45000/ 45600/45636/FINAL_PKG_FHWA-JPO-12-042_V3.pdf. 2011. 
18. University of Buffalo, see http://www.civil.buffalo.edu/research/ research-areas/area:transportation-systems-engineering/ section:projects/project:an-evaluation-of-likely-environmentalbenefits-of-lowest-fuel-consumption-route-guidance-in-thebuffalo-niagara-metropolitan-area/. 2014.

19. Barth M, Boriboonsomsin K. Energy and emissions impacts of a freeway-based dynamic eco-driving system. Transp Res D Environ 14. Elsevier Press; 2009. pp. 400-10.

20. Xia H, Boriboonsomsin K, Barth M. Dynamic eco-driving for signalized arterial corridors and its indirect network-wide energy/ emissions benefits. J Intell Transp Syst Technol Plann Oper. 2013;17(1). doi:10.1080/15472450.2012.712494.

21. U.S. Department of Transportation. AERIS-applications for the environment: real-time information synthesis: eco-signal operations modeling report, publication number FHWA-JPO-14-185, see http://ntl.bts.gov/lib/54000/54900/54930/Eco-Signal_Operations_ Modeling_Report__Final_508_-_012615.pdf. 2014.

22. U.S. Department of Transportation. AERIS-applications for the environment: real-time information synthesis: eco-lanes operational scenario modeling report, publication number FHWA-JPO-14186, see http://ntl.bts.gov/lib/54000/54900/54929/Eco-Lanes_ Modeling_Report_-_Final_508_-_012615.pdf. 2014.
23. [WG4CEM, 2013] EU Working Group for Clean and Efficient Mobility, see http://www.imobilitysupport.eu/library/imobilityforum/working-groups/active/ict-for-clean-and-efficient-mobility/ reports-4/2332-wg4cem-final-report-131308.

24. Cooperative mobility systems and services for energy efficiency, see http://www.ecomove-project.eu/. 2015.

25. ECOSTAND: Joint EU-Japan-US task force on the development of a standard methodology for determining the impacts of ITS on energy efficiency and $\mathrm{CO}_{2}$ emissions, see http://www.ecostandproject.eu/. 2015.

26. EU Compass4D research project, see http://www.compass4d.eu/. 2015.

27. Co-operative systems for sustainable mobility and energy efficiency, see http://www.cosmo-project.eu/. 2015.

28. Co-operative networked concept for emission responsive traffic operations, see http://www.traffictechnologytoday.com/news.php? NewsID=27116. 2014.

29. Assessment Methodologies for ICT in multimodal transport from user behaviour to CO2 reduction, see http://www.amitran.eu/. 2015.

30. Cooperative advanced driver assistance system for green cars, see http://www.transport-research.info/web/projects/project_details. cfm?id=44395. 2015 . 